\title{
Función docente ideal/real en las representaciones sociales del profesorado
}

The Ideal/Real Role of the Teacher in the Social Representation of Teaching

Artículo de investigación | Research article

Fecha de recepción: 31 de julio de 2018 Fecha de aceptación: 05 de noviembre de 2019

Fecha de disponibilidad en línea: mayo de 2021

doi: 10.11144/Javeriana.m14.fdir

\section{Carlos Vecina-Merchante} carlos.vecina@uib.es

Universidad Nacional de Educación a Distancia, España (iD) ORCID: http://orcid.org/0000-0001-8439-8455

Sonsoles San Román-Gago

s.sanroman@uam.es

Universidad Autónoma de Madrid, España

ORCID: http://orcid.org/0000-0003-3114-4720

Para citar este artículo | To cite this article

Vecina-Merchante, C. \& San Román-Gago, S. (2021). Función docente ideal/real en las representaciones sociales del profesorado. magis, Revista Internacional de Investigación en Educación, 14, 1-27. doi: 10.11144/Javeriana.m14.fdir 


\title{
Resumen
}

Esta investigación analiza representaciones sociales del profesorado y su función, para conocer si existen discursos justificativos y cuál es su estructura. Emplea una metodología cualitativa de técnicas de análisis del discurso y grupos de discusión. Los resultados indican tres representaciones sobre su función docente ideal, contrapuestas a las representaciones sobre las que se materializa la práctica, que van desde una tipología de docentes de la ortodoxia instrumental hasta el aperturismo vital del alumnado. Como conclusión, se plantea que es necesario considerar esa diversidad interiorizada en el profesorado, tanto en el diseño como en la implementación de las políticas educativas.

\section{Palabras clave}

Orientación; orientación pedagógica; profesor de secundaria; sociología

\begin{abstract}
This research analyses the social representations of teachers and their role to know if there are justifying discourses and their structure. With a qualitative methodology, discourse analysis and group discussion techniques. Results show three representations about their ideal role as teachers against the ones which relate to the actual ones materialized in the practice. A typology of teachers from instrumental orthodoxy to the vital openness of students. In conclusion, it is necessary to consider that internalized diversity in teachers, both in the design and implementation of educational policies.
\end{abstract}

\section{Keywords}

Counselling; educational guidance; secondary school teachers; sociology 
Descripción del artículo | Article description

Este artículo forma parte de la investigación realizada en el proyecto I+D +i Contexto socioeconómico y orientación educativa y profesional del profesorado de secundaria, aprobado por el Ministerio de Economía y Competitividad (España), con referencia CSO2013-47168-R, bajo la dirección de la investigadora principal, Sonsoles San Román-Gago.

\section{Introducción}

Las transformaciones sociales plantean nuevos retos a las instituciones educativas (Fernández-Enguita, 2007), cuya relación con la promoción del desarrollo económico, social y personal está en el punto de mira, lo que genera una crisis de la tradicional concepción de la institución escolar (Dubet, 2010) e implica ampliar los objetivos educativos y considerar la formación permanente a lo largo de toda la vida (Castells, 2006). La sociedad global lleva aparejada la exigencia a los centros de enseñanza de nuevas funciones sociales; esto incide en el profesorado y su práctica educativa (Marchesi, 2005) y en la percepción que se tiene sobre su papel en la sociedad (Bolívar, 2004). La búsqueda del buen profesor se convierte en un objetivo desde diferentes instituciones y debates, tanto nacionales como internacionales (Marina, 2015). La educación y el profesorado están en el punto de mira de las políticas educativas, y las directrices que llegan a España desde la Unión Europea apuntan en esa dirección, planteando retos como el aprendizaje permanente, la calidad educativa, la cohesión social, la ciudadanía activa y potenciar el incremento de la creatividad y la motivación (Red Española de Información sobre Educación, 2018), siguiendo así concepciones de la United Nations Educational, Scientific and Cultural Organization (Unesco) (2004) de lo que debe representar la educación para la sociedad actual y para un futuro de cohesión e integración social.

En este escenario, resulta interesante aproximarnos a lo que el profesorado de Educación Secundaria Obligatoria (ESO) entiende y tiene interiorizado sobre aquello que debería ser su función como transmisor de cultura y de hábitos y como agente socializador de un alumnado que está a punto de decidir su trayectoria, y continuar un itinerario académico, profesional o desmarcarse de la educación formal para entrar en el mercado de trabajo. Nos interesa, por tanto, destacar las representaciones sociales que aparecen en torno a esa cuestión, pensando en la necesidad de acercarse a la concreción, al día a día de la práctica educativa en el aula, como una forma de mostrar aquello que el profesorado tiene interiorizado, un conocimiento 
que puede ser útil a la hora de pensar en qué educación se quiere y qué papel debe, puede o está dispuesto a asumir el profesorado. Dando por hecho que no existe un discurso, ni una posición homogénea, nos interesa descubrir qué ocurre en la praxis, cómo se la representa, si se asemeja a la representación de lo que se considera su función o, por el contrario, dista de ella, y es vista como un ideal, o cómo se ubican los elementos periféricos en el discurso. Para esto nos referimos a los recursos discursivos, a la retórica usada por el profesorado para justificar la acción docente que desarrolla.

Con este fin se ha utilizado una metodología cualitativa para el análisis del discurso docente. La hipótesis de partida es la existencia de diferentes modelos de orientación del profesorado, los cuales responden a las representaciones sociales sobre su función docente, el tipo de cultura y aquello que pretenden transmitir, y se relacionan, en el discurso justificativo de dicha acción, con elementos condicionantes y fines de la acción social y educativa, dependiendo del modelo interiorizado por cada sujeto. Como fin último, se pretende que este trabajo sea útil en el proceso de concienciación del profesorado, de su papel, de los modelos sociológicos que rigen su acción y de las consecuencias que de ellos pueden derivarse.

\section{Función docente como función profesional y social}

El profesorado podría mantener una representación de lo que sería el buen maestro vinculada al compromiso de la profesión y a su responsabilidad: un buen maestro es un profesional "dotado de pensamiento crítico y reflexivo [...], motivado por las funciones igualitarias y democráticas de la educación, preocupado por construir una escuela democrática e inclusiva" (Usategui \& Del Valle, 2012, pp. 192-196). Marina (2015) postula que el buen profesor se hace a sí mismo, y a esto puede ayudar la formación continua y el trabajo colaborativo con compañeros e investigadores. Este autor cita estudios realizados por medio de observación en el aula, como el "proyecto de investigación Measures of Effective Teaching, cuyo fin es buscar claves de la buena docencia y de cómo mejorar la educación" (Marina, 2015, p. 97), pero en el caso español considera que es muy difícil seguir esas metodologías, por considerarse intrusivas en el aula y por el escaso uso que de ellas se hace. La capacidad de innovar está condicionada por el coste emocional de cambiar la metodología, las competencias profesionales interiorizadas y un compromiso profesional influido negativamente por las reformas educativas, debido a la falta de participación real de los docentes y las pocas expectativas que les generan. Frente a este inmovilismo se precisan "programas formativos que promuevan la autonomía, el 
ajuste situacional y la protección emocional" (Monereo, 2010, p. 584). Sin embargo, este inmovilismo está marcado por las continuas reformas educativas y por las incertidumbres generadas entre el profesorado, por la falta de recursos, por la ambigüedad de su papel y por la incertidumbre de las directrices a seguir en su práctica docente: "Si son precisos cambios en la profesionalidad para hacer frente a los desafíos del trabajo docente [...], no pueden ser invocados sólo para ponerse al servicio de las nuevas políticas" (Bolívar, 2004, p. 20).

En España, se mantuvo un inmovilismo legislador en materia educativa hasta que en 1970 la Ley General de Educación sustituyó a la Ley Reguladora de la Enseñanza, que databa de 1857, de esta forma se asentó una estructura básica del sistema en diferentes niveles e itinerarios, la cual se mantuvo hasta la Ley Orgánica General del Sistema Educativo (LOGSE, 1990), cuando la enseñanza obligatoria se extendió hasta los 16 años. En la actualidad rige la Ley Orgánica para la Mejora de la Calidad Educativa (LOMCE, 2013), una norma que no ha estado exenta de fuertes polémicas y protestas por parte de las familias y del profesorado, englobadas en la Marea Verde por una Educación Pública de Calidad, una huelga general de la enseñanza pública, a la que se sumó la recogida de 1,7 millones de firmas, contra la LOMCE, antes de su aprobación (Rogero-García, Fernández \& Ibáñez, 2014). Junto a esas reformas se ha ido definiendo el papel docente, sus funciones y su papel como orientador.

Se entiende por orientación escolar aquella orientación académica, psicopedagógica y profesional que el alumnado, tal como establecía la LOGSE, en su artículo 60, debe recibir con respecto a sus distintas opciones educativas y a la transición del sistema educativo al mundo laboral, prestando singular atención a la superación de determinantes sociales discriminatorios que condicionan el acceso a los diferentes estudios y profesiones. Este principio del sistema educativo, introducido en España por la Ley General de Educación de 1970, adquirió una mayor presencia y se convirtió en una clave fundamental de la actividad y la calidad educativa, posteriormente más desarrollada en la Ley Orgánica de Educación (LOE) de 2006, que formalizó su presencia en los distintos planos de la actividad educativa, como parte de una educación integral. Los cambios legislativos inciden en la concepción de la función educativa y docente, trastocando también el marco de la orientación. La LOMCE establece una rigidez en las posibilidades de elección y cambio de los itinerarios académicos, determinando la trayectoria académica del alumnado e implicando también directamente a los tutores e indirectamente al profesorado en su acción para orientar a los alumnos en la elección de las materias troncales de opción (art. 25.9). Asimismo, el cambio en las asignaturas y su relevancia, la reforma de la formación 
profesional, las reválidas y las formas de acceso a la universidad pueden generar incertidumbre en el profesorado a la hora de acompañar al alumnado en su proceso de aprendizaje y en las injerencias que pueda ejercer en este.

La diversidad cultural de las aulas es otro elemento más de incertidumbre, ante el que el profesorado vive con temor de que "resulte imposible mantener el carácter cultural predominante" (Suárez-Orozco \& SuárezOrozco, 2003, p. 101). En el fondo se encuentra la búsqueda de un sentido a su profesión, libre de la amenaza de la fractura entre su ética profesional, la institución educativa y una sociedad en continuo y profundo cambio a la que dar respuesta. Estas presiones llegan desde diferentes frentes, como es el caso de las que se emiten en torno a las directrices y objetivos de la estrategia española y europea 2020, con su énfasis en la mejora de los resultados académicos, la reducción del abandono escolar temprano y el destacado papel que debe cumplir la educación en las sociedades modernas (Instituto de Evaluación, 2011). Asimismo los continuos cambios legislativos se ven reflejados en las directrices que se van marcando a los centros y al profesorado desde cada consejería de educación del territorio en el espacio regional' (Doncel, San Román \& Vecina, 2019).

El profesorado podría encontrarse en una encrucijada marcada por una autorrepresentación negativa, al no poder asumir los cambios que la sociedad va experimentando y aquellos que implican una respuesta vehiculada a través de su función educadora, lo que se ha denominado choque entre dos identidades, la institucional y la profesional. Ahora se demanda una pedagogía adaptada a cada alumno, acompañamiento, adaptación curricular, motivar, relacionarse y compartir el proceso educativo con la familia, etc. Pero el problema subyace en la falta de normas y reglas claras en torno a ese nuevo papel docente, lo que lo lleva a justificar el fracaso por la influencia de otros agentes, como la familia, la sociedad, la legislación, las características socioeconómicas, etc. (Bolívar, 2004). La brecha entre teoría y práctica docente se justifica donde hay riesgo de poner en cuestión el trabajo educativo del profesorado. La teoría tiende a aparecer en el discurso docente para hacer referencia a la necesidad de facilitar la inserción social y cultural, pero pocas veces se materializa (García, 2003): "Se tiende a hacer énfasis en los factores externos que no ponen en duda la eficiencia y eficacia del profesorado" (Colectivo loé, 2007, p. 240), e incluso se muestra a este como una víctima pasiva de una situación motivada por agentes externos "de una sociedad que no los valora" (Marchesi, 2005, p. 175).

1 En España, el territorio se encuentra dividido administrativamente en 17 comunidades autónomas, que a su vez cuentan con consejerías de Educación, con potestad para desarrollar la legislación educativa estatal, con medidas concretas en su territorio. 
Este estudio pretende adentrarse en la estructura de las representaciones sociales del profesorado sobre su función docente, los puntos clave que se ubican en su núcleo y aquellos que lo hacen en la periferia y qué poder tiene cada uno de estos en dichas representaciones y en su propio discurso. Nos interesa constatar la distancia entre las representaciones de lo ideal y de lo real, este último elemento igualmente subjetivo, pues no se ha desarrollado una observación en el aula para corroborar lo que allí acontece, sino que surge del propio discurso docente y de la posición que ocupa en este, por lo que se constata en la periferia de la estructura representacional y la forma que adquiere, siguiendo así a Abric (2001) cuando describe el papel de la periferia y su relación con aquello que definen como real los sujetos: "La realidad [...], lo vivido por el sujeto" (p. 24).

Interesa analizar si ocurre la confluencia entre ambas dimensiones o si se abre una brecha entre el ideal teórico que el profesorado tiene interiorizado sobre su función educativa y la representación de una realidad manifiesta que ocurre en la práctica diaria del aula. En definitiva, se quiere ver si existen posicionamientos teórico-ideales distintos, en qué modelo se ubica el profesorado y, dependiendo de este, qué funciones asume como inherentes a su labor y qué elementos dan consistencia al discurso justificativo de su acción.

\section{Representaciones sociales de la función docente: ideal vs realidad}

Las representaciones sociales facilitan la ordenación, la percepción y la acción frente a un ente externo, variable o desconocido. Se constituyen en forma de esquema, lo que facilita la organización del universo que nos rodea: "Obra de sujetos sociales deseosos de conferir un sentido a los acontecimientos" (Moscovici, 1975, p. 75). Ellas son una forma de conocimiento social para mantener una posición frente a determinados acontecimientos $u$ objetos, y se conciben no solo como una forma de entender "una realidad", sino también de comunicarla: "Un conocimiento socialmente elaborado y compartido" (Jodelet, 1988, p. 473).

La aproximación a la educación desde este paradigma tiene gran presencia en investigaciones realizadas en Latinoamérica, y "se considera que en la presente década se incrementará el número de trabajos basados en esta teoría" (Piña \& Cuevas, 2004, p. 102). De las diversas líneas de aproximación al concepto de las representaciones sociales, en este estudio nos aproximamos al ámbito educativo desde una doble perspectiva, con un planteamiento cercano al de Jean Claude Abric y su teoría del núcleo central, sin dejar de lado una aproximación global más sociológica del discurso 
y a la vez concreta, "una perspectiva etnográfica [...] para enriquecer el desarrollo teórico [...]: la utilización de la entrevista [...], el trabajo con grupos [...], inferir la estructura representacional" (Perera, 2003, pp. 15-16), una perspectiva cercana a la de Denise Jodelet. La teoría del núcleo central anuncia que la organización de una representación, además de estar jerarquizada, se encuentra ordenada en torno a un núcleo central y una zona periférica (Abric, 1976). A este respecto, Rodríguez (2007) apuesta por metodologías cualitativas para identificar las representaciones y su estructura.

El núcleo es unificador, constituye el elemento estable de la representación y su resistencia al cambio, y el resto de elementos se encuentran relacionados con él, pero son más o menos susceptibles al cambio, dependiendo de ítems que llegan de nuevas experiencias. Es la frecuencia de aparición en el discurso, e incluso la cantidad de lazos con el conjunto, lo que indica la centralidad de los elementos (Abric, 2001, p. 22): "Dimensiones socioafectivas, sociales o ideológicas [...]: una norma, un estereotipo, una actitud fuertemente marcada estarán en el centro de la representación".

Los elementos periféricos contienen informaciones seleccionadas e interpretadas, juicios de valor, estereotipos y creencias. Estos se encuentran jerarquizados y orbitan en torno al núcleo, ilustrando y justificando la significación de la representación; hablan de la experiencia; dependen del contexto; resultan del anclaje de la representación, y son a la vez defensores del núcleo, pero también susceptibles a un cambio en el contexto (Abric, 2001; Rodríguez, 2007): "Constituyen la interface entre el núcleo y la situación concreta, en la que se elabora o funciona la representación [...] en la realidad [...]. Hablan del presente y de lo vivido por el sujeto" (Abric, 2001, pp. 23-24).

Nos encontramos así con un doble sistema: primero, el central, determinado socialmente, relacionado con factores históricos, sociológicos e ideológicos, y asociado a valores y principios. En este artículo este sistema sería la forma que adquiere en el discurso el ideal de la función docente, y observaremos la forma en que se posicionan los discursos a través de los elementos periféricos, para cada uno de los núcleos o representaciones sociales de su función. La periferia discursiva justifica un ideal y la práctica real como representación social de lo que se define como el trabajo docente en el aula del día a día. En segundo lugar, se encuentra el sistema periférico, que permite adaptaciones individuales y contextuales de cada individuo, en función de su propia experiencia y de cómo esta es vivida (Abric, 2001).

Las representaciones influyen en el profesorado, en la autoconciencia del papel social de cada uno y de aquello sobre lo que puede actuar con éxito (Vecina, 2016), además transmiten percepciones de la realidad, influyendo así en su entorno (Chaib, Danermark \& Selander, 2012; Uzzell \& Blud, 
1993). Las representaciones se consideran un factor inherente a la función docente, explicitadas en su práctica y en su discurso. El profesorado analiza de forma distinta su acción y aquellos factores implicados en el proceso de enseñanza-aprendizaje. Este hecho permite establecer una tipología.

Hay docentes que reflexionan con más asiduidad sobre las limitaciones y obstáculos a superar en su trabajo, aunque surgen diferencias entre el colectivo, pues si bien algunos consideran un elevado número de variables que influyen en esa función, otros se centran en aspectos de planificación y didáctica, obviando lo que sería una práctica educativa integral. En un extremo contrario se ubican aquellos que no suelen plantearse esas cuestiones, y únicamente lo hacen cuando son conscientes de que comenten algún error: "Los profesores hacen evidente la ausencia de reflexión en su práctica, pues la conciben como un elemento que existe fuera de ellos" (Chehaybar, 2006, p. 237).

La concepción de la orientación holística se considera una acción realizada junto al proceso educativo, con la finalidad del desarrollo personal, social y profesional del sujeto, un trabajo que no depende únicamente del orientador, sino de la totalidad de agentes educativos y sociales ( $\mathrm{Pa}$ rras, Madrigal, Redondo, Vale \& Navarro, 2009). El profesorado vincula su función indirectamente con una forma de orientación educativa, a veces sutil, que se traduce en la práctica orientando el acompañamiento del alumnado en su proceso de aprendizaje (Villar, San Román \& Hernández i Dobon, 2018).

Llegamos así a encontrar una tipología de modos de representar el hecho de ejercer la docencia que nos permite comprender desde qué perspectiva proyecta, orienta o focaliza... Hablamos de modelos de orientación educativa y profesional. Las representaciones que utilizan los individuos están relacionadas con la posición que ocupan y el habitus que los constituye (Bourdieu, 1996). Cuando definimos la función docente, la enmarcamos en las formas que adquiere la interacción docente-discente como habitus de transmisión cultural, de entender o de poner en práctica dicha acción. De ese análisis se identifica una gradación ascendente: instrumental, emotivasituacional y de aperturismo vital, en función de lo que cada forma implica en su intención, práctica educativa, contenido y del efecto que espera generar en el alumnado (San Román \& Vecina, 2017).

\section{Metodología}

La investigación cualitativa resulta apropiada para indagar en la comprensión de la realidad, vista desde sus protagonistas y en el análisis de representaciones sociales. De ahí que hayamos optado por los grupos de 
discusión como una técnica apropiada para fomentar el debate y extraer un discurso generado en el contexto en el que desarrollan su acción, como representación de una estructura social y cultural subyacente a este (Del Val \& Gutiérrez, 2006). El análisis discursivo se ha realizado teniendo en cuenta elementos hermenéuticos, con el fin de descubrir significados, interpretar palabras y textos y aproximarse a la comprensión de la realidad vista y vivida por sus protagonistas (Martínez, 2015). Dicho método resulta útil para el análisis de representaciones sociales en el caso de la búsqueda de su estructura núcleo-periferia. El análisis de los textos facilita un encuadre de los elementos que las constituyen: de los centrales, al considerar su repetición, incidencia y por la metáfora que encierran, y de los periféricos, como aproximaciones justificativas (Rodríguez, 2007). Siguiendo a Rodríguez, se ha procedido a identificar elementos principales del discurso y la posición con respecto a estos, de los que forma parte aquello que ella misma denomina la retórica discursiva, englobando así a diferentes elementos que se ubicarían en el núcleo o en la periferia de este, dependiendo de su centralidad en la representación. Así pues, la base teórico-analítica de este trabajo tiene como referentes la teoría del núcleo de Abric y su vinculación con el análisis cualitativo de Rodríguez. De esta aproximación se derivan las diferentes categorías identificadas.

Para realizar el análisis de textos se contó con el soporte del programa informático NVivo-QSR 12. Con respecto a los elementos del núcleo, se circunscriben a este directamente por la forma teórico-metodológica que adquiere la definición de la función docente como representación hecha por el profesorado. La tipología elaborada en estudios anteriores (San Román \& Vecina, 2018) sirvió para agrupar los contenidos de dichas definiciones: 1) instrumental, 2) emotiva-situacional y 3) de aperturismo vital. Los elementos periféricos se asociaron a cada núcleo por su significatividad en el discurso docente, tanto por la constante alusión que se hacía a estos como por ser utilizados a la hora de justificar los factores que inciden en la función docente y la comprensión de la realidad que tiene el profesorado, constatada en su campo de representación. Estos elementos aparecieron también en un formato trivial en otras investigaciones, en las que se agrupaban elementos discursivos en torno a una interrelación entre sociedad, familia, profesorado y alumnado (San Román \& Vecina, 2017), pero en este caso el interés está en ver cómo se relacionan en el discurso con la tipología de representaciones en torno a la función docente.

Se llevó a cabo una aproximación al concepto de representaciones y los factores que podrían formar parte de su configuración en el discurso del profesorado a partir del análisis de 10 grupos de discusión, uno por cada comunidad autónoma (CCAA) objeto de estudio (con entre 6 y 8 
participantes por grupo). La composición siguió criterios de heterogeneidad, con el fin de que hubiera una representación de las principales características del profesorado: sexo, edad, años de experiencia, materias de las que eran docentes, ejercer la docencia en $3 .^{\circ}$ o $4 .^{\circ}$ de ESO y por último pertenecer a centros de enseñanza pública o privada.

Se seleccionó una muestra intencional de $10 \mathrm{CCAA}^{2}$, siguiendo criterios del contexto socioeconómico y del sector productivo, así: Andalucía y Murcia (primario $>8 \%$ ), Castilla y León, Navarra, La Rioja, País Vasco y Valencia (industrial 15\%), Madrid, Baleares y Canarias (servicios > $80 \%$ ) (Instituto Nacional de Estadística, 2013); bajo la idea de tener una amplia representación de realidades y territorios del Estado español, asegurando así una mayor riqueza discursiva. Se da así por válida la premisa de que la biografía de cada uno y el contexto determinan las representaciones sociales y la propia praxis educativa que se desarrolla (Jodelet, 1988). Si bien el objeto de este trabajo no es desentrañar la existencia de diferencias entre CCAA (ese será el objeto de trabajos posteriores, en los que se ha aplicado una encuesta a todo el territorio del Estado), en este caso se ha pretendido acceder a la máxima diversidad de situaciones, teniendo en cuenta la heterogeneidad de realidades socioeconómicas, políticas y culturales de las diferentes CCAA, con el fin de poder acceder a una mayor riqueza discursiva dentro de las posibilidades de un estudio cualitativo.

\section{Análisis de resultados}

En el análisis de los discursos del profesorado y sus representaciones sociales ha aparecido una doble alusión a la función docente: por una parte, aquella que el profesorado tiene idealizada y, por otra, la que considera que ocurre en la práctica, junto a esto se encuentran también una serie de factores asociados, argumentativos justificativos de una u otra opción, ubicados en la periferia de dicha representación.

\section{La función docente vista desde el profesorado}

Cuando al profesorado se le pregunta directamente sobre su función, expresa una definición relacionada con orientación, dirección, acompañamiento, etc., y a medida que profundiza en su discurso aparecen otras cuestiones vinculadas a esta y a los factores con los que se relaciona. Empieza a discernir entre el ideal (la definición que el profesorado hace de su función, entendiendo que formaría parte del imaginario social sobre su papel como

2 Las comunidades autónomas son una división político-administrativa, con transferencias de gestión política. 
docente) y el real, el discurso periférico del campo de representación que permite justificar su acción en el aula (lo que dice que hace o se ve obligado a hacer por las dificultades a las que se enfrenta) ${ }^{3}$, es decir, por un lado, aquello que es teórica y políticamente correcto, implicando a su forma de actuar, de enseñar, de difundir, etc., y, por otro, lo que considera que pasa en realidad. Aquí entramos en un juego discursivo en el que el docente justifica lo que hace (lo real), pero también podemos identificar la representación de lo que oficialmente, profesionalmente, socialmente, se espera de su trabajo y la representación que tiene sobre otros aspectos, aquellos que le sirven como justificación de lo que hace ${ }^{4}$. Se trata de ir viendo cómo se sitúa cada factor en torno a lo que debería ser el núcleo de la representación sobre su función (las definiciones que asocian a lo esta que debería ser) y los elementos que formarían la periferia, entendidos como aquellos que aparecen de forma significativa en su discurso, como una alusión predominante en su retórica.

Así, en ese esfuerzo discursivo por definir su práctica educativa, el profesorado se mueve en una doble dimensión ideal-real, entre aquello que debería ser el ideal de su acción, de su papel docente, y lo que representa como práctica real. Si bien ambas no dejan de ser al fin y al cabo representaciones sociales de su función: la ideal vinculada a lo que el profesorado supone que la sociedad espera de su papel social, pedagógico y educativo, mientras que la denominada "real" no es más que una justificación de su papel, en muchas ocasiones alejado de los cánones esperados, de lo políticamente correcto:

Se pone mucho énfasis en eso, en el sentido no de dirigirles de una manera determinada, ni de intentar moldearles, sino orientarles para que desarrollen sus propias potencialidades, las competencias famosas. [...] Luego es cuando te das de bruces con la realidad (BIL, §5) .

3 Un ejemplo: cuando el profesorado define su función como dirigir, coordinar el contenido que han de asimilar, ser fiel a un currículum se está refiriendo a un ideal, pues inmediatamente justifica que eso no ocurre así, sino que se encuentra con factores condicionantes, como, por ejemplo, un alumnado con bajo nivel o sin habilidades para atender en una clase. En este caso está narrando su visión sobre la realidad (una representación) de lo que es su función en la práctica diaria.

4 Lo que dice hacer no deja de ser también un elemento subjetivo de su propio discurso, una representación al fin y al cabo, a través de la cual justifica esa oposición entre ideal y real.

5 Estos acrónimos corresponden a la denominación con la que se ha definido el grupo al que pertenece la cita, los números se refieren al párrafo o párrafos en los que aparecen en la transcripción de dicho grupo de discusión. 


\section{Finalidad del profesorado ideal vs real}

A continuación se profundiza en la forma que adquiere el discurso entre lo que el profesorado representa que debería ser su función ideal y cómo la define en la práctica ${ }^{6}$.

\section{Dimensión ideal}

En el discurso más concreto y trivial sobre la función docente pueden identificarse caracteres susceptibles de ser clasificados en torno a tres tipos de funciones: instrumental, emotiva-situacional y de aperturismo vocacional. A continuación, se presenta una breve aproximación a cada una de estas:

a) Instrumental: centrada en un contenido académico curricular, con un planteamiento más directivo; el docente aparece como transmisor del saber "institucional": "Nuestra finalidad sería dirigirlos: [...] este es el tema, leéroslo antes" (BIL, §§ 133-136).

b) Emotiva-situacional: es una posición intermedia, ubicada entre la instrumentalización y la interiorización de valores en el alumnado. Se trataría de la representación social de una función que va más allá de ceñirse a la trasmisión de contenido curricular, el docente es una herramienta para motivar, la innovación pedagógica es lo que le ayuda a educar. Motiva para favorecer la asimilación de los contenidos. El docente es en sí una herramienta que debe cuidar a través de la formación y la innovación: "El objetivo de la enseñanza no puede ser homogéneo, porque el grupo es muy heterogéneo [...], [sino] volver a plantearnos las cosas como si fuéramos maestros de Primaria" (MUR, §§ 778-786).

c) De aperturismo vital: es la consideración de una función que va más allá de puros contenidos académicos, el ideal de la acción docente sería interiorizar en el alumnado una forma de ser, un modelo cultural flexible, capaz de adaptar el saber a todas las situaciones, formar ciudadanos para el futuro incierto, pero preparados para hacerle frente, siempre con valores coherentes y racionales: "No dirigirles, ni intentar moldearles, orientarles para que desarrollen sus potencialidades" (BIL, §§ 158-161); "Dotar de habilidades y estrategias para que tengan sentido crítico, sean capaces de emprender, tomar decisiones y abordar cualquier profesión" (VAL, §§ 214-220).

6 La definición en sí misma es pura representación de su papel en la sociedad, no tanto como ideal, sino cómo es identificado por el profesorado, una acción vinculada a un contexto complejo de múltiples influencias.

7 Tipología a la que se llegó en estudios previos, realizados con estos mismos grupos de discusión (San Román \& Vecina, 2018). 
Dimensión real

En el análisis de los textos, llama la atención un discurso dicotómico: el profesorado define su función desde una visión de lo ideal, para seguidamente justificar su declaración, aunque ese recurso varía con base en el tipo de representación que tienen sobre dicho papel. Así, en el caso del tipo instrumental, se refiere, inmediatamente en contraposición a la representación sobre lo real, a aquello que considera que ocurre y le impide poder desarrollar su trabajo, por lo que su definición inmediatamente se convierte en un ideal. Podemos observar un gradual en la justificación, yendo de más a menos en su justificación ideal vs real, siguiendo la línea del tipo de función más cerrada a más abierta. En este último extremo se situaría el tipo 3, el aperturismo vital. Encontramos, por tanto, diferentes posiciones justificativas que aparecen vinculadas a la tipología de las representaciones sociales sobre su función docente ideal.

\section{Tipo 1. Función instrumental}

El discurso marca una clara diferencia entre lo ideal y lo real. La contradicción está vinculada con el tipo de alumnado, su familia y la sociedad, vista como un ente negativo que carga sobre el profesorado unas determinadas funciones, siendo juzgado por el grado de cumplimiento de estas. Sin embargo, el profesorado considera que estas no le corresponden y que van en detrimento del objetivo de su labor docente, que no es otra que transmitir el conocimiento curricular. De este modo, centra en estos elementos la justificación de los posibles malos resultados del alumnado.

Si ponemos el punto de mira en los elementos estructurales de la representación social, podemos identificar su núcleo en la función docente y en la forma que se presenta, en este caso, instrumental, y cómo el discurso va presentando elementos justificativos, que aparecen a modo de contradicciones. En el discurso se establece una presencia lineal de cuatro factores: profesorado, alumnado, familia y sociedad.

Figura 1

Retórica discursiva y elementos periféricos en el tipo 1 (dimensión real)

\begin{tabular}{|l|l|l|l|}
\hline \multicolumn{4}{|c|}{ Distancia de los elementos periféricos en la representación } \\
social de la función docente ideal \\
\hline
\end{tabular}

Fuente: elaboración propia 
En la función instrumental (figura 1), el periférico de primer orden es el alumnado. Este es el elemento más próximo al núcleo de la representación. Se presenta como el objeto del trabajo docente, pero distorsionado y lejos de un ideal. Impide directamente la eficacia de la transmisión educativa. El tipo de alumnado determina el éxito o el fracaso docente. La distancia cultural, a modo de habitus, marca una brecha y una determinación para poder ejercer o no una práctica educativa coherente con los objetivos de este tipo 1. El alumnado es presentado como problema: "Tienes que empezar a explicar desde cero y al día siguiente vuelves a empezar" (BIL, §§ 137-138), "Se convierte en dirigir porque no puedes hacer otra cosa" (BIL, § 149), "Cuando se pone un examen hay que estudiarlo, hay que ponerlo [...]. Has de entender que no te estamos enseñando esto por puro deporte, sino para tu formación" (MAD, §§ 261-263), "El objetivo de lo público es la socialización y dentro de eso, lograr la máxima excelencia posible. Y esa excelencia muchas veces no se logra porque es inviable" (PAM, §§ 571-573).

El periférico de segundo orden es la familia. Este es un sujeto sistémico en el que está inserto el alumnado. Se presenta como un agente de socialización contradictorio con la transmisión escolar y distorsionante y falto de competencias parentales, educativas, culturales, etc.:

Es que no saben una frase [...], y a lo mejor los padres tienen parte de la culpa [...]. Tienes que escuchar a tus hijos, tienes que estar con ellos, pero se ponen muchas excusas en las familias. [...] El profesor ha cogido, ha asumido ese papel de tener que estar pendiente de ellos, pero es una locura [...]. Eso no nos lo dice la Ley [...]. No está escrito, pero nos vemos obligados a hacerlo (MUR, §§ 934-947).

El periférico de tercer orden es la sociedad en general, que se presenta como un espacio en el que todos los agentes están ubicados e influidos por ella. Es vista como un contexto de transformación social irracional, se ubica al margen de la institución escolar, a la que deriva funciones sociales que se contradicen con las del profesorado. Se convierte en un agente extraño más destructivo que constructivo, influye en la familia y desorienta su papel educador, incide en la escuela y deriva funciones inasumibles, sin directrices claras, recursos ni sentido para poder darles respuesta desde esta institución:

No hay que pensarla de una manera tan [...] que toda la sociedad depende de nosotros [...]. Vamos cargados con responsabilidades que alguien ha soltado. Alguien ha liberado responsabilidades en ayuntamientos, en autonomías y familias y las coge el profesor (MUR, §§ 878-920).

Hemos asumido, yo creo, demasiada responsabilidad. Y a la escuela se le ha metido absolutamente todo. No es solamente que tú des lengua, sino 
tienes que conseguir que el niño esté callado, que esté sentado, que te responda [...]. Entonces, hay problemas de género, y se mete un problema de género en la escuela; hay problemas de educación vial, y se mete educación vial [...]. Todo va a la escuela (GRA, §§ 199-206).

\section{Tipo 2. Función intermedia emotiva-situacional}

Esta función es motivadora e innovadora. Se centra más en una autocrítica, en su obsesión por innovar, aprender, echar de menos metodologías que le permitan obtener lo mejor del alumnado, para motivarlo al estudio. Fija la mirada de contraposición en la legislación, por las dificultades que le impone y la formación docente, como un recurso para poder ser más eficientes y eficaces en su cometido. El alumnado es un medio y un fin: un medio para ejercer su profesión docente, pues es el objeto de esta, y un fin en el sentido de que será sobre quien se produzca el efecto educador.

La función ideal aparece en una posición intermedia entre lo ideal y lo real. La figura docente es clave, pues aparece en un primer lugar en cuanto a proximidad con el núcleo de la representación social. Parte del discurso está constituido por una autocrítica hacia el mismo profesorado, que se ve a sí mismo como una herramienta destinada a mejorar el aprendizaje del alumnado; esto se consigue con la motivación y con el hecho de ayudar a despertar el interés por el conocimiento (esta sería una de las principales tareas). La formación docente, la innovación, la búsqueda de estrategias pedagógicas aparecen constantemente en sus definiciones. El discurso no entra en una contradicción directa, más bien se muestra una reflexión de búsqueda constante de aquello que debe ser la función docente, una finalidad que precisa estar continuamente innovando y buscando formas de adaptarse a una realidad cambiante. No es un discurso tan negativo como en el tipo 1, aunque sí lo es cuando se habla de la administración, la legislación o el contenido y las exigencias del currículum. Esto se ve como el principal inconveniente para desarrollar su función docente.

Figura 2

Retórica discursiva y elementos periféricos en el tipo 2 (emotivo-situacional)

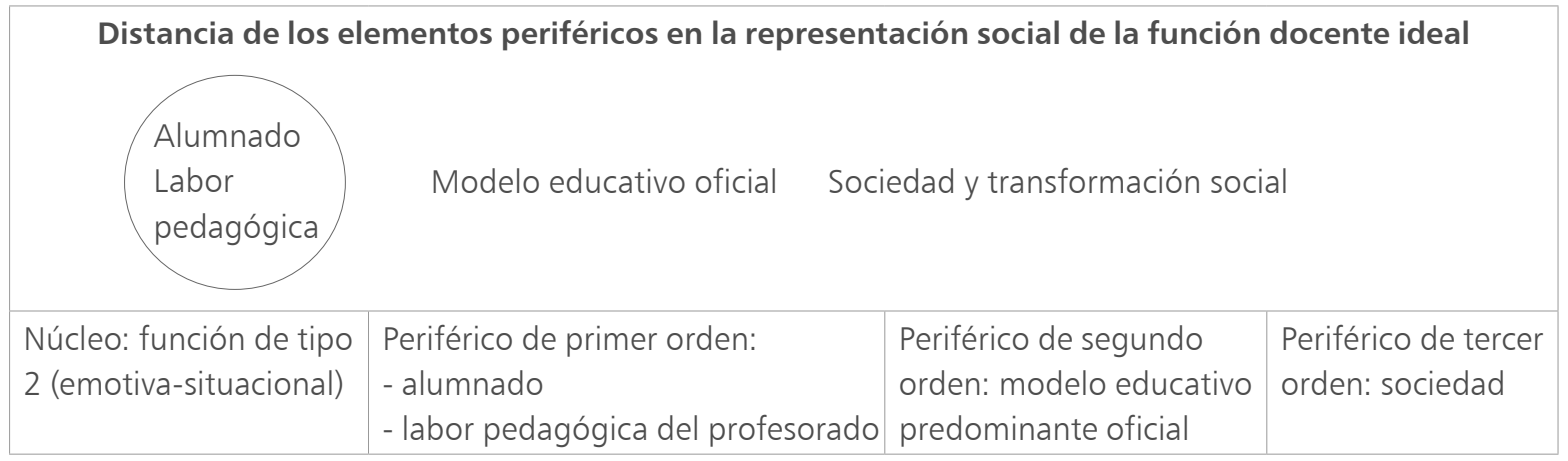

Fuente: elaboración propia 
En la función emotivo-situacional (figura 2), el periférico de primer orden es el alumnado y la labor pedagógica, que aparecen en el mismo lugar y directamente relacionados. El primero no aparece como un gran problema de difícil solución y un obstáculo para la función docente, sino más bien como un reto educativo, es el objeto sobre el que el docente debe trabajar su profesionalidad. De ahí que la acción pedagógica se encuentre en el mismo lugar, pues es aquello con lo que ha de contar el docente en su trabajo. La motivación del alumnado aparece en un lugar destacado:

Lo que hice con algunos chicos que tenían problemas [...], yo lo que intento en la clase es que los chicos se lo pasen bien, que hagan cosas que les gustan. Pero siempre trabajando con el currículum (TEN, §§ 559-563).

En la ESO sobre todo porque les estamos obligando a estar y no es una enseñanza optativa. Yo creo que hay que hacer un planteamiento de que lo importante es el desarrollo integral del alumno (MUR, §§ 788-790).

Ganándote al disruptivo, favoreciendo momentos de encuentro y de escucha, eres capaz de que se vuelva a enganchar a lo académico (RIO, $\S \S 948-950)$.

¿Quién soy yo y qué pinto yo aquí? ¿Qué tengo que hacer? Esa gran pregunta la pueden ir sacando desde las matemáticas [...]. Yo creo que por todas las asignaturas, no importa qué seas (GRA, §§ 795-799).

Yo creo que somos eso, diseñadores de estrategias para que el alumnado aprenda (GRA, §§ 214-215).

El periférico de segundo orden es el modelo educativo oficial, el cual, si bien aparece en segundo orden, es el primero en tanto condicionante, en este caso negativo. Se ubica aquí porque el profesorado presenta un discurso constructivo, por lo que en este, el alumnado y la acción pedagógica ocupan una posición predominante, como justificación de su labor hacia el ideal. En el modelo educativo oficial se incluyen la legislación, la administración, el currículum y una dura crítica hacia estos elementos por dificultar la acción docente y poder tener éxito en su trabajo: "El problema empieza ya en el modelo. O sea, las condiciones en las que estamos" (BIL, §§ 237-238), "Creo que son tantas funciones para las cuales no estamos preparados, falta una directriz que enseñe a hacer estas cosas" (PAM, §§ 497-498).

En el periférico de tercer orden se alude a un ente macrosociológico, en ocasiones citado como el contexto socioeconómico, el modelo de sociedad dominante, el cual parece ejercer una presión más general y externa a la institución escolar, pero que determina aquello a lo que la escuela y la labor docente deben responder. No se presenta de una forma totalmente negativa, sino más bien como unas necesidades asumidas y vistas como retos educativos. 


\section{Tipo 3. Función de aperturismo vital}

Esta función va más allá del currículum, pues el profesorado se ve a sí mismo como un modelo a seguir, son quienes tienen la llave de la transformación social. Su discurso no es ya un enfrentamiento ideal-real, sino más bien un discurso optimista, seguro de su capacidad para influir en el alumnado y la sociedad. Estos factores aparecen como retos frente a los que trabajar. La sociedad aparece, en cierta medida, como opositora a su capacidad transformadora y los modelos que transmite la escuela, a pesar de ello, el profesorado confía en sus posibilidades y la función que realiza. Es una idea romántica, en la que se recupera la educación como vía para la liberación, frente a los modelos sociales contradictorios con la escuela. Cree en la educación por encima de todo, le otorga un valor que va más allá de una relación funcionalista, la educación no solo ha de significar una relación directa con el mercado de trabajo, sino algo más, como formar en la integridad humana.

La realidad está formada por las contradicciones y problemas sociales diversos, en un contexto en el que priman unos valores distintos a los que la escuela ha de transmitir. Pero esa realidad no gana la partida, es un elemento de gran envergadura e influencia, aunque el profesorado confía en la educación y su capacidad para crear un alumnado íntegro y con capacidad crítica. El discurso transita por concepciones cercanas a la pedagogía o a la escuela activa, en la que el alumnado es diverso y se debe trabajar a partir de sus inquietudes y particularidades.

Figura 3

Retórica discursiva y elementos periféricos en el tipo 3 (aperturismo vital)

\begin{tabular}{|c|c|c|c|}
\hline & Alumn & 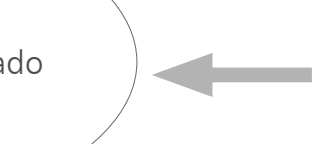 & $\begin{array}{l}\text { Sociedad } \\
\text { Modelo educativo oficial } \\
\text { amilia }\end{array}$ \\
\hline $\begin{array}{l}\text { Núcleo: función de tipo } \\
3 \text { (de aperturismo vital) }\end{array}$ & $\begin{array}{l}\text { Periférico de primer orden: } \\
\text { - educación } \\
\text { - profesorado }\end{array}$ & $\begin{array}{l}\text { Periférico de segundo } \\
\text { orden: alumnado }\end{array}$ & $\begin{array}{l}\text { Periférico de tercer orden: } \\
\text { - sociedad } \\
\text { - modelo educativo } \\
\text { predominante oficial } \\
\text { - familia }\end{array}$ \\
\hline
\end{tabular}

Nota: la distancia de los elementos periféricos en la representación social de la función docente ideal muestra que no existe una separación con respecto al núcleo; es un discurso retórico en el que los elementos se ubican con respecto a una distancia del centro, aunque sí queda fuera de ese círculo el periférico de tercer orden: la sociedad, el modelo oficial y la familia, que son de influencia, pero la acción del profesorado tiene una misión de contrarrestar, y no forma parte de esos círculos concéntricos. 
En la función de aperturismo vital (figura 3), en el periférico de primer orden se ubica al profesorado y la educación, por su responsabilidad y ética profesional, cuya función debe responder a los modelos dominantes en la sociedad, los cuales tienden a ser contrarios al que transmite la escuela. Entre estos mensajes negativos se encuentran valores dominantes como el individualismo, el dinero y el éxito rápido, la intolerancia, etc., frente a los cuales la escuela transmite cooperativismo, solidaridad, democracia, educación y conocimiento:

Yo creo que los sistemas educativos, tal como están y las leyes que salen, las leyes son papeles. Entonces, los que producen los cambios son los profesores y las profesoras [...]. Somos ahora educadores, no solamente damos materia en secundaria, sino tenemos que ir mucho más allá. Y educar [...] el desarrollo integral de las personas (PAMPL, §§ 97-104).

Estamos en una sociedad donde, bueno, pues todos sabemos cómo está la cosa, y qué padres tienen algunos [...]. Entonces que el profesor sea un referente de honradez, dicho con mayúsculas (RIO, §§ 1167-1170).

El periférico de segundo orden es el alumnado, con sus particularidades y posibilidades en potencia. Ya no se habla de un único alumnado, como un conjunto homogéneo o dicotómico bueno-malo, sino que ahora se habla de diversidad, de capacidades múltiples ante las que el profesorado tiene un campo abierto para explorar y educar:

Claro, es que cada crío es un mundo y es complicado trabajar con ellos y que saquen lo que llevan dentro [...]. No es cuestión de darles unos contenidos y "que sepan esto y ya está"; sino el mundo es diverso y se van a enfrentar a situaciones muy diversas y tienen que saber desarrollarse, 0 ponerse delante de una situación (VAL, §§ 263-269).

La vida que tienen en el barrio ese es una vida [...] todo el día callejeando, con un montón de problemas, no tienen familia, hay droga [...]. Que se sientan seguros y que sientan que existe algo más, que no solo es aquello que tienen, que si estudian, pues a lo mejor la meritocracia no acierta siempre, pero por lo menos les va a abrir la cabeza y les va a servir para algo (TEN, §§ 572-578).

El periférico de tercer orden son los valores dominantes en la sociedad y aspectos legislativos o institucionales que representan rigidez en la acción. Aquí aparecen el currículum, la legislación educativa, pero también la sociedad en su conjunto. Muchas veces vistas como inconvenientes, aunque 
el profesorado habla en su discurso de acción frente a todo ello. Siente que tiene un papel destacado como educador, una nueva tarea que la sociedad le encomienda, ante las dificultades sociales y la incapacidad de que otras instituciones puedan asumir ese papel:

Tengo que hacer que estudien una serie de cosas porque el currículum me obliga y les tengo que poner una nota. Pero mi objetivo es que no odien la asignatura [...] y que les guste pensar y que [...] después, cuando abandonen el centro, les pueda servir para la vida. Y que sean críticos, que sean autónomos, no sean manipulables por cualquiera y que tengan un filtro en el cerebro para que puedan tener una vida autónoma (PM, $\S \S 759-766)$.

Nos encontramos aquí con el final de la búsqueda de una retórica discursiva perfecta, caracterizada por la integración de los elementos periféricos de lo "real" en la representación ideal de la función docente; los participantes no lo plantean como una distancia dicotómica entre "lo que se debería hacer y lo que ocurre", sino como elementos que forman un conjunto. En el tipo 1 se muestran claramente los cortes y distinciones en el discurso, se trata de una trama lineal. El tipo 2 es menos lineal y más sinérgica y retórica; sin embargo, en el tipo 3 se hace difícil separar los elementos periféricos, si bien se identifican y tienen mayor o menor importancia con respecto a su cercanía e influencia sobre el núcleo. Ahora todo es un conjunto, es decir que se trata de una visión de la función docente que abarca todo, tiene claro los límites y las potencialidades, y el resto de los factores forman parte del contexto en el que se actúa. El fin es la transformación o la preparación del alumnado para que sean personas íntegras y críticas y se asegure así un futuro coherente con la educación que se pretende.

Para concluir, el discurso es susceptible de ser vinculado con una estructura en dos dimensiones: socioafectivas y sociales o ideológicas, las cuales se encontrarían de forma transversal e inherentes a la relación entre el tipo de representación social de la función docente y la distancia que el sujeto considera en la dicotomía ideal-real, pues parece existir una vinculación afectiva, emocional, entre lo que considera su función ideal, el fundamento de esta y lo que influye o desvía su acción. A modo de síntesis, dicha relación y estructura se puede observar en la tabla 1. 
Tabla 1

Tipologías y dimensiones ideológica y socioafectiva

\begin{tabular}{|l|l|}
\hline $\begin{array}{l}\text { Dimensión ideológica } \\
\text { (representación de lo ideal) }\end{array}$ & $\begin{array}{l}\text { Dimensión socioafectiva } \\
\text { (representación de lo real) }\end{array}$ \\
\hline $\begin{array}{l}\text { Tipo 1. Instrumental: dirigir al alumnado, indicar qué } \\
\text { estudiar, dotar de competencias teórico-curriculares. } \\
\text { Docente asimilacionista. }\end{array}$ & $\begin{array}{l}\text { Afectos negativos hacia los elementos considerados } \\
\text { más significativos en ejercer una acción para } \\
\text { desvirtuar su función ideal. }\end{array}$ \\
\hline $\begin{array}{l}\text { Tipo 2. Emotivo-situacional: despertar curiosidad, } \\
\text { motivación, docente como herramienta pedagógica. } \\
\text { Docente innovador. }\end{array}$ & $\begin{array}{l}\text { Afectos vinculados con la superación, con la } \\
\text { búsqueda de mejoras a través de la innovación } \\
\text { pedagógica. }\end{array}$ \\
\hline $\begin{array}{l}\text { Tipo 3. De aperturismo vital: desarrollar } \\
\text { competencias, enfrentarse a la vida, favorecer visión } \\
\text { crítica de la realidad. Docente innovador y optimista } \\
\text { hacia la educación. }\end{array}$ & $\begin{array}{l}\text { Afectos neutros hacia los elementos perturbadores y } \\
\text { positivos hacia la educación como herramienta para } \\
\text { el cambio. }\end{array}$ \\
\hline
\end{tabular}

Fuente: elaboración propia

\section{Discusión}

El análisis del discurso docente y el campo de representación consecuente han sido claves en la identificación del núcleo y la periferia. Tal y como lo plantea Abric (2001), esta última es un recurso para justificar al primero. La retórica utilizada es diferente para cada uno de los tipos de representación sobre la función docente identificados, lo que implica por tanto diferentes aproximaciones justificativas (experiencias de la realidad, para Abric), utilizando para ello otros tantos recursos discursivos, coincidiendo en este sentido con los planteamientos de los autores de referencia que se han seguido en este trabajo (Abric, 2001; Martínez, 2015).

Hay, por tanto, diferencias entre el profesorado en la forma de asumir los nuevos roles que la sociedad reclama (aprendizaje permanente, calidad educativa, cohesión social, ciudadanía activa y potenciamiento del incremento de la creatividad y la motivación). En un principio, pudiera parecer que el profesorado de tipo instrumental es el más acorde con las directrices que los profesores reciben desde el currículum y las exigencias académicas formales derivadas de la legislación educativa y las concreciones que marcarían las diferentes consejerías de educación, como el entorno más próximo que concreta el currículum, el contenido evaluativo, etc. (Doncel et al., 2019). Si bien el profesorado del tipo instrumental mantiene que su función es regirse por el currículum académico y los resultados de adquisición del proceso educativo del alumnado, lo cierto es que este tipo se encontraría en una situación y perspectiva clásica, sin dar respuesta a las nuevas demandas. Sería el tipo aperturista vital el que estaría respondiendo 
con mayor énfasis a esas cuestiones, tales como potenciar el incremento creativo, la cohesión social y, en definitiva, crear ciudadanos para afrontar los retos de la sociedad actual y futura. Este tipo de profesorado piensa que la educación es una herramienta adecuada, mientras que el tipo emotivosituacional se encontraría en el camino para lograrlo, pues ya responde a algunas de las cuestiones.

Es evidente que las representaciones parten del contexto en el que se ubican los sujetos y son una forma de comunicar la realidad (Jodelet, 1988). Los participantes en el estudio usan las representaciones sociales para ubicar su función en el universo educativo que les envuelve, se trata de un recurso que les ayuda a ordenar ese maremágnum en el que se mueve el sistema educativo actual en España, un contexto en el que la función docente y la escuela se encuentran en el centro de un debate sobre su aporte y capacidad para dar respuesta a la sociedad de la información y el conocimiento, utilizando para ello instituciones arraigadas aún en los modelos de enseñanza del siglo $X X$; el reto consiste en que sean capaces de abrirse a las exigencias de esa nueva realidad (Marina, 2015), en la que incluso se habla del declive de la institución educativa como tal (Dubet, 2010). Las representaciones han jugado un papel destacado en la ordenación del universo incierto (Moscovici, 1975), y transmiten percepciones de la realidad que influyen en la acción que se desarrolla (Chaib et al., 2012). Este hecho ha facilitado ordenar los discursos en un argumento coherente, de modo que se diferencie qué elementos aparecen como periferia del tema central y cómo se posicionan, siguiendo así un orden establecido del discurso, lo cual aparece en su retórica y en el propio pensamiento de los sujetos, según su experiencia y cómo sea vivida (Abric, 2001; Rodríguez, 2007).

El profesorado tiene claro su papel y la forma que tendría el buen maestro (Usategui \& Del Valle, 2012). Otra cosa es la forma que debería tomar este como ideal. Es aquí donde surgen las diferencias explicitadas en la tipología (instrumental, emotiva-situacional y de aperturismo vital). De igual forma, esas diferencias muestran formas distintas de adaptarse a las exigencias actuales y los papeles a asumir en la función docente. Puede que haya incertidumbre y ambigüedad en el papel a cumplir, como plantea Bolívar (2004), y que buena parte de la culpa esté en el continuo ir y venir de sucesivos cambios legislativos que no acaban de acertar, al no contar con una participación efectiva del profesorado (Monereo, 2010), y que posiblemente esa situación lleve a este a ir más allá del escaso movimiento que permiten los resultados académicos a lograr con su alumnado, aunque también es cierto que se han identificado formas distintas de hacer, de ser profesor, lo que es acorde con la forma de interpretar y asumir el papel docente, con planteamientos teóricos, pero también vitales. 


\section{Conclusiones}

El discurso del profesorado estudiado se mueve en una doble dimensión: 1) la ideal, centrada en aquello que se considera es lo políticamente correcto, para lo que debe ir encaminada su función y práctica educativa; 2) la real, definida por lo que el profesorado opina (representa) que pasa en el día a día del aula y los elementos que inciden para poder desarrollar su función ideal. Esta última dimensión aparece en el discurso como el elemento periférico, ante el contraste entre el ideal representado y la representación de la realidad de la función docente.

La heterogeneidad del profesorado se encuentra distribuida en tres tipos de definiciones, tres representaciones sociales sobre su función: 1) instrumental, centrada en un contenido académico curricular, con un planteamiento más directivo, en la cual el docente aparece como un transmisor del saber "institucional"; 2) emotiva-situacional, que es una posición intermedia en la que el docente se ve a sí mismo como una herramienta, un elemento motivador para el estudio, y 3 ) de aperturismo vital, en la cual el profesorado representa su función vinculada con una acción liberadora y crítica, de construir adultos del futuro capaces de dar respuesta a los retos de una sociedad incierta.

Cuando se pregunta al profesorado sobre su función, este utiliza un recurso retórico dicotómico, y en la dimensión ideal expresa representaciones sobre su función, pero a continuación justifica con una representación de lo que es la realidad en el ámbito educativo y los elementos que lo configuran (comunidad educativa con las familias, compañeros, la legislación educativa y sus directrices, el alumnado, etc.). Este recurso dicotómico que justifica al final lo que en realidad hacen o piensan en su práctica diaria se ha encontrado que no es el mismo según la tipología de docente. Un tipo instrumental diferencia notablemente un discurso sobre el ideal y sobre el real; la contradicción se centra en el alumnado, la familia, la sociedad y las características que dificultan el hecho de cumplir con su función como docentes. En el tipo emotivo-situacional es la función motivadora e innovadora, su obsesión por innovar y formarse para dar respuesta curricular, a partir de la motivación del alumnado, un hecho que le lleva a una autocrítica de sus capacidades; la mirada está centrada en la legislación, las trabas administrativas y la formación docente, el alumnado no es el problema, sino más bien un medio sobre el que ejercer su profesión, para dar respuesta a los cambios sociales y las nuevas exigencias curriculares. El tipo aperturista vital se ve a sí mismo como un modelo a seguir por los alumnos, es la llave socializadora y educativa; es optimista y cree en sus capacidades; la transformación social no es un problema sino un reto a su trabajo; en parte tiene 
un fondo romántico en el que la educación se muestra como la liberación de los adultos del futuro, y el discurso sobre la realidad está constituido por contradicciones y problemas sociales a los que hacer frente, pero no es catastrofista, pues confía en la educación para ganar la partida a los valores contrarios a los que se enseñan en la escuela.

La búsqueda de una escuela capaz de responder a los nuevos retos de la sociedad debe contar con el profesorado como agente educativo de relación directa con el alumnado. Pero no solo eso, sino que también se precisa una coherencia entre lo que unos y otros, profesorado y administración, entienden por las funciones del primero y el camino a seguir por su orientación académica. En caso contrario, resulta difícil avanzar hacia una educación integral y profunda. Ir más allá del currículum académico no puede ser un elemento que dependa de la concepción particular que tenga cada profesor o profesora de la finalidad de su trabajo educativo ni significar tener que ir más allá de ese currículum para conseguir una educación más amplia; aunque el problema subyace también en los aspectos teórico-sociales interiorizados en el profesorado, su papel y la finalidad de la propia escuela. Tal vez este hecho lleve a plantearse retos para la configuración de una profesión que afecten a la misma formación del profesorado, como el espacio académico en el que este inicia un proceso formal como futuro profesional.

\section{Sobre los autores}

Carlos Vecina-Merchante con su tesis doctoral sobre representaciones sociales del profesorado inició una línea de investigación que le ha llevado a diversas publicaciones nacionales e internacionales sobre la función docente y la interacción con el alumnado. Actualmente se centra en dos vertientes: por un lado, el ámbito de intervención comunitaria y los procesos de educación no reglada y, por otro, la educación reglada con la identificación de los elementos sociales que condicionan la orientación docente.

Sonsoles San Román-Gago es pionera en el estudio de los procesos de feminización docente, con reconocimiento nacional e internacional. Ha publicado cinco libros en editoriales nacionales e internacionales y también numerosos artículos. Su línea de investigación se dirige ahora hacia el estudio de las representaciones sociales y culturales para identificar los modelos de orientación desplegados y practicados por los docentes, atendiendo a las características y condiciones socioeconómicas y culturales del entorno en el territorio español.

\section{Referencias}


Abric, J. C. (1976). Jeux, Conflicts et représentations sociales (tesis de Estado). Universidad de Provence, Aix-en-Provence, Francia.

Abric, J. C. (2001). Las representaciones sociales: aspectos teóricos. En J. C. Abric (Dir.), Prácticas sociales y representaciones (pp. 11-32). México: Coyoacán.

Bolívar, A. (2004). La educación secundaria obligatoria en España. En la búsqueda de una inestable identidad. Educación Secundaria, 2(1), 1-23. Recuperado de https://revistas.uam.es/index.php/reice/article/view/5534

Bourdieu, P. (1996). Cosas dichas. Barcelona: Gedisa.

Castells, M. (2006). La sociedad red. Madrid: Alianza.

Chaib, M., Danermark, B. \& Selander, S. (2012). Education Professionalization and Social Representations. On the Transformation of Social Knowledge. Nueva York: Routledge.

Chehaybar, E. (2006). La percepción que tienen los profesores de educación media superior y superior sobre su formación y su práctica docente. Revista Latinoamericana de Estudios Educativos, 336(3-4), 219-259. Recuperado de https://dialnet.unirioja.es/servlet/articulo?codigo $=2363520$

Colectivo loé. (2007). Inmigración, género y escuela. Exploración de los discursos del profesorado y del alumnado. Madrid: CIDE, Ministerio de Educación y Ciencia, Gobierno de España.

Del Val, C. \& Gutiérrez, J. (2006). Prácticas para la comprensión de la realidad social. Madrid: McGraw-Hill.

Doncel, D., San Román, S. \& Vecina, C. (2019). Orientación académica y profesional: percepción del mercado de trabajo desde las consejerías de Educación españolas. Educação e Pesquisa, 45, 1-23. Recuperado de https://www. scielo.br/pdf/ep/v45/1517-9702-ep-45-e188063.pdf

Dubet, F. (2010). Crisis de la transmisión y declive de la institución. Política y Sociedad, 47(2), 15-25. Recuperado de https://revistas.ucm.es/index.php/ POSO/article/view/POSO1010230015A

Fernández-Enguita, M. (2007). Educar es cosa de todos: escuela, familia y comunidad. En J. Garreta (Ed.), La relación familia-escuela (pp. 13-32). Lleida: Edicions de la Universitat de Lleida.

García, A. (2003). Identidad y cultura: efectos en la educación intercultural. Revista Interuniversitaria, 10, 253-264. Recuperado de https://www.redalyc. org/articulo.oa?id=135015168013

Instituto de Evaluación. (2011). Objetivos educativos europeos y españoles. Estrategia Educación y Formación 2020. Madrid: Ministerio de Educación.

Instituto Nacional de Estadística (INE). (2013). Encuesta de Población Activa. Madrid: Autor, Administración General del Estado.

Jodelet, D. (1988). La representación social: fenómenos, concepto y teoría. En S. Moscovici, Psicología social II. Pensamiento y vida social (pp. 469-493). Barcelona: Paidós.

Marchesi, A. (2005). Qué será de nosotros, los malos alumnos. Madrid: Alianza. Marina, J. A. (2015). Despertad al diplodocus. Barcelona: Ariel.

Martínez, M. (2015). Hermenéutica y análisis del discurso como método de investigación social. Paradigma, 23(1), 9-30. Recuperado de http://prof.usb. ve/miguelm/hermenyanalisisdisc.html

Monereo, C. (2010). iSaquen el libro de texto! Resistencia, obstáculos y alternativas en la formación de los docentes para el cambio educativo. Revista de 
Educación, 352, 583-597. Recuperado de https://dialnet.unirioja.es/servlet/ articulo?codigo $=3224456$

Moscovici, S. (1975). Introducción a la psicología social. Barcelona: Planeta.

Nussbaum, M. \& Sen, A. (1993). La calidad de vida. México: Fondo de Cultura Económica.

Parras, A., Madrigal, A. M., Redondo, S., Vale, P. \& Navarro, E. (2009). Orientación educativa: fundamentos teóricos, modelos institucionales y nuevas perspectivas. Madrid: Centro de Investigación y Documentación Educativa (CIDE), Ministerio de Educación, Gobierno de España.

Perera, M. (2003). A propósito de las representaciones sociales: apuntes teóricos, trayectoria y actualidad. La Habana: CIPS.

Piña, J. M. \& Cuevas, Y. (2004). La teoría de las representaciones sociales. Su uso en la investigación educativa en México. Perfiles Educativos, 25(106), 102-124. Recuperado de http://www.scielo.org.mx/scielo.php?script=sci_ar ttext\&pid=S0185-26982004000100005

Red Española de Información sobre Educación (Redie). (2018). Marco estratégico Educación y Formación 2010. Madrid: Ministerio de Educación y Formación Profesional, Gobierno de España.

Rodríguez, T. (2007). Sobre el estudio cualitativo de la estructura de las representaciones sociales. En T. Rodríguez \& M. L. García (Coords.), Representaciones sociales. Teoría e investigación (pp. 157-190). Guadalajara: CUCSH-UDG.

Rogero-García, J., Fernández, C. J. \& Ibáñez, R. (2014). La "Marea Verde". Balance de una movilización inconclusa. RASE, 7(3), 567-586. Recuperado de https://www.google.com/url?sa $=t \& r c t=j \& q=\& e s r c=s \&$ source $=$ web\&cd $=\& v e d=2$ ahUKEwiclMaysazqAhVKUt8KHQtsBolQFjAAegQIBBAB\&url=ht tps\%3A\%2F\%2Fdialnet.unirioja.es\%2Fdescarga\%2Farticulo\%2F5017885. pdf\&usg=AOvVaw24JXnpzWT88uj_om78U3sL

San Román, S. \& Vecina, C. (2018). Habitus del profesorado de $3^{\circ}$ y $4^{\circ}$ de la ESO y su relación con los modelos de orientación educativa y profesional en el ámbito de las CCAA en el territorio español. Ponencia presentada en la Il Conferencia Ibérica de Sociología de la Educación. Córdoba: Federación Española de Sociología, Associaçao Portuguesa de Sociologia y Universidad de Córdoba.

Suárez-Orozco, C. \& Suárez-Orozco, M. M. (2003). La infancia de la inmigración. Madrid: Morata.

Usategi, E. \& Del Valle, A. I. (2012). Aprender a formar. Vitoria-Gasteiz: Fundación Fernando Buesa Blanco Fundazioa.

United Nations Educational, Scientific and Cultural Organization (Unesco). (2004). Apprendre pour le travail, la citoyenneté et la durabilité. París: Autor. Recuperado de https://unevoc.unesco.org/fileadmin/user_upload/pubs/ Flyer-07f-Sustainable.pdf

Uzzell, D. \& Blud, L. (1993): Vikings! Children's Social Representations of History. En G. Breakwell \& D. Canter, Empirical Approaches to Social Representations (pp. 110-133). Oxford: Clarendon.

Vecina, C. (2016). Las representaciones sociales sobre el rol de cada uno en la comunidad, una barrera para la participación y el trabajo conjunto. Trabajo 
Social Global, 6(10), 3-26. Recuperado de https://dialnet.unirioja.es/servlet/ articulo?codigo $=5564856$

Vecina, C. \& Giró, J. (2018). Representaciones sociales, profesorado y familia. Ehquidad Internacional Welfare Policies and Social Work Journal, 10, 67-88. Recuperado de https://doi.org/10.15257/ehquidad.2018.0009

Villar, A., San Román, S. \& Hernández i Dobon, F. J. (2018). Niveles de conciencia del profesorado en su función orientadora: una tipología enmarcada en la lógica difusa. Educaçao e Pesquisa, 44, 1-17. Recuperado de https://www. redalyc.org/articulo.oa? $\mathrm{id}=29858802071$ 